\title{
Medical Image of the Week: Mediastinal Metastases Causing Right Ventricular Outflow Obstruction
}

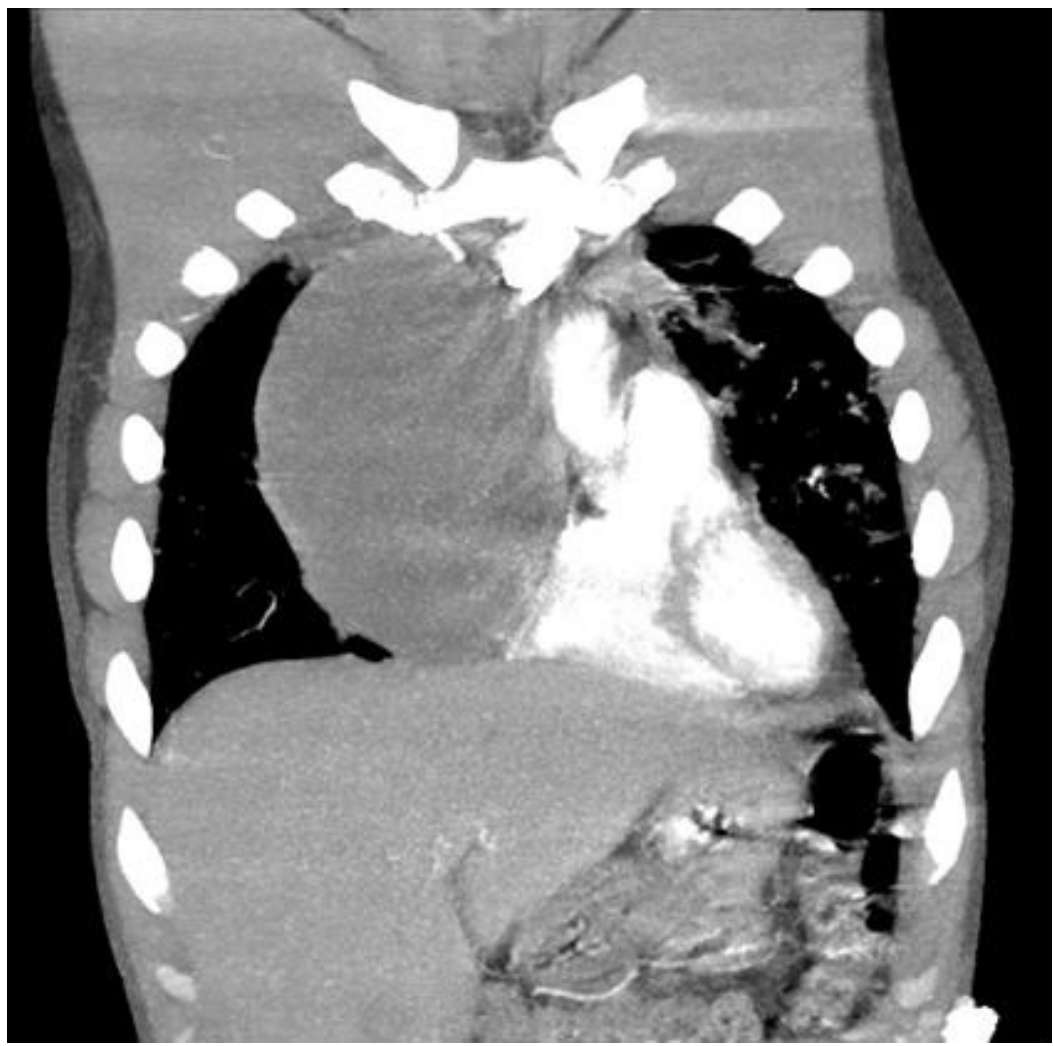

Figure 1. Computed tomography (CT) of chest showed large right mediastinal mass (arrow) causing mass effect on the heart.

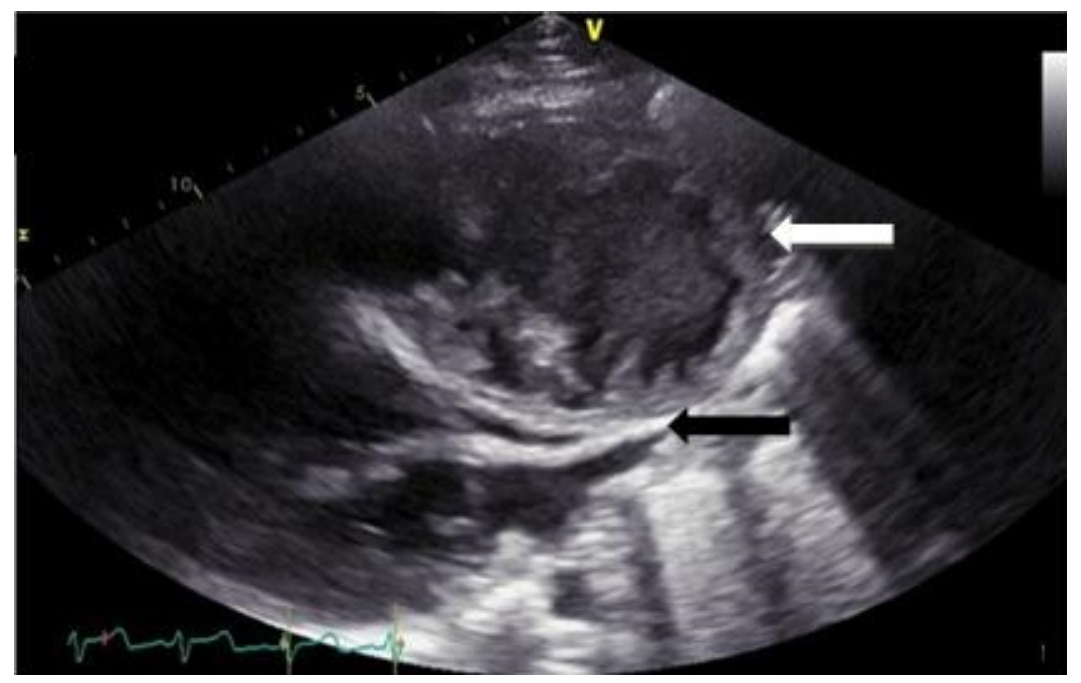

Figure 2. Echocardiography showing large extra-cardiac mass (white arrow) compressing on right ventricle and its outflow tract (black arrow). 
A 36-year-old man with a history of testicular choriocarcinoma with metastases to the lung presented with a 2-days history of hemoptysis. Initial diagnosis of the malignancy was made about 5 months earlier and he was treated with platinum based chemotherapy with a partial response.

He reported two days of significant hemoptysis, associated with shortness of breath and pleuritic chest pain and rapidly developed acute hypoxic respiratory failure requiring emergent intubation and mechanical ventilation. Computed tomography (CT) of chest showed large right mediastinal mass with diffuse reticular and nodular opacities predominantly in the left lung (Figure 1).

A pulmonary angiogram was performed that showed multiple active bleeding sites in the bronchial arterial system. These were treated with embolization. He developed shock and during investigations the echocardiogram showed a significant compression of the superior vena cava, right atrium and right ventricle by the malignant mass (Figure 2). Despite aggressive therapy and resuscitative therapies he continued to deteriorate and did not survive the hospital stay.

Mediastinal tumors are a rare cause of extrinsic right ventricular outflow tract (RVOT) obstruction. Echocardiography is an important tool in the assessment of hemodynamic effects caused due to such pathology including degree of compression and pressure gradients.

Kai Rou Tey $\mathrm{MD}^{1}$, Bhupinder Natt MD²

${ }^{1}$ Department of Internal Medicine, University of Arizona College of MedicineSouth Campus, Tucson, AZ USA

${ }^{2}$ Division of Pulmonary, Critical Care, Allergy and Sleep, University of Arizona Medical Center, Tucson, AZ USA 